\title{
EDITORIAL
}

\section{Parent, child and public involvement in child health research: core value not just an optional extra}

\begin{abstract}
Pediatric Research (2019) 85:2-3; https://doi.org/10.1038/s41390018-0245-z
\end{abstract}

\section{THE RIGHTS OF THE CHILD}

Healthcare professionals are advocates for families and children but have no consistent framework research involvement. The Convention on the Rights of the Child (CRC) enshrined 'The right to an opinion' stating that all children have the right to express their views. They also have the right to be informed and give their opinion about the world around them ${ }^{1}$. Here we discuss PatientPublic Involvement (PPI) in research and why it is important and provide a framework to assist healthcare professionals.

\section{WHAT IS PPI}

Patients' and public involvement (PPI) (https://involvementmapping.patientfocusedmedicine.org) means involving anyone not professionally interested or experienced in health and care in in research. Public involvement, $\mathrm{PI}$, is another term often used which describes initiatives to give lay people an effective, active role in health and care research. PPI and PI have similar goals: to develop research that addresses patients' and the public's needs, and thereby improve the success, cost-effectiveness and impact of research. Furthermore, close involvement of patients and the public can facilitate rapid dissemination and implementation of research findings.

Patient and public (and parent) involvement in medical research differs considerably between countries, as well as between patient groups, medical specialties and institutions. The use of different terminology and definitions in different countries has contributed to this variation. ${ }^{2}$ Similar types of patient and family involvement are termed engagement in North America and participation in the Netherlands whereas the UK National institute for Health Research (NIHR) divides different activities into engagement, involvement and participation depending on their nature (https://www.nihr.ac. uk/patients-and-public/). Involving children research adds another layer of complexity as terminology needs to include infants, children, teenagers and young adults as well as their families.

Organisations and initiatives such as iCAn (International Children Advisory Network: a global consortium of Youth Advisory Groups) and NIHR Generation R are dedicated to providing a voice for children and families in paediatric medicine and research, and provide resources, advice and examples effective PPI. ${ }^{3}$

\section{WHY IS IT ESSENTIAL...WHAT IS THE EVIDENCE}

Advocacy and patient participation are becoming increasingly integrated in child health research and practice as healthcare moves to embrace family-centred models of care. This, and the move towards meaningful PPI in research more generally, has highlighted the high degree to which such children and their families would like to be involved in future research. During the creation of the Royal College of Paediatrics and Child Health $(\mathrm{RCPCH})$ research charter, children and their families ${ }^{4}$ clearly indicated that they wanted to participate in the planning, design and implementation of research projects. Furthermore, there is good evidence that research that integrates meaningful PPIresearch performed with or by the research participants rather than on them-leads to tangible benefits for researchers ranging from better trial recruitment and retention (http://www.jla.nihr.ac. uk/about-the-james-lind-alliance/) to enhanced research impact.

\section{HOW TO DO THIS?}

Developing partnership between families, children, the public and healthcare workers is essential across the research process, from research priority setting, clinical trial design, ethics, medical publishing, medical conferences, through to policy.

There are paediatric and neonatal examples of high quality PPI that cover the different stages of the research cycle. Following a framework developed by the James Lind Alliance (http://www. comet-initiative.org/), parents, patients, and the public have been involved in identifying priorities to be addressed in future preterm birth research. In relation to trial design, the Core Outcome Measures in Effectiveness Trials (COMET) Initiative has developed a comprehensive handbook to guide researchers seeking to develop core outcome sets for use across clinical trials, a key component of which is parent and patient involvement. ${ }^{5,} 6$ Research funders are increasingly supporting these initiatives, for example the primary funder of medical research in the United Kingdom, the NIHR, mandates reference to both core outcome sets and James Lind Alliance priority setting partnerships where they exist, when funding clinical trials.

Meaningful parent, patient and public involvement is increasingly required when publishing research. Medical journals are increasingly modifying their editorial policies to recognise the importance of PPI-the British Medical Journal's campaign for patient-partnership is an excellent example of collaboration in this area. $^{6}$ In Paediatric Research we have started this journey by introducing parent perspectives to complement papers published in the journal. Another suggestion is the inclusion of a lay summary in all manuscripts to improve accessibility. Medical conferences are also changing to recognise and value PPI such as the Cochrane colloquium (https://colloquium.cochrane.org/ patients-included) to ensure there is a patient in the programme, on the stage and in the audience. The European Foundation for the Care of Newborn Infants has developed a guide for parents on research which provides guidance on meaningful engagement of families in research projects ${ }^{7}$

Financial and educational resources are needed to allow researchers, families and children to engage in collaborative research. Funding mechanism exist from the NIHR and Wellcome Trust in the UK as well as Patient-Centered Outcomes Research Institute (PCORl; https://www.pcori.org/) in the USA which provide support to researchers and families engaging in PPI research. 
Resources such as guidance notes, and practical advice on costing and publication libraries have been established by the NIHR the INVOLVE group(http://www.invo.org.uk/).

As paediatricians, neonatologists and researchers in child health we work closely with parents, patients and families routinely and continuously to provide the optimal care for all children, and are privileged to be allowed fulfil this role. High quality evidence suggests that applying this approach to academic paediatrics and neonatology leads to better, more applicable and impactful research. The benefits to children and their families from participation in research projects may be augmented by involvement in all aspects of the project from its inception to publication and implementation, and be a source of empowerment.

Eleanor J. Molloy ${ }^{1,2,3,4}$, Silke Mader ${ }^{5}$, Neena Modi ${ }^{6}$ and Chris Gale ${ }^{6}$

${ }^{1}$ Paediatrics, Academic Centre, Tallaght University Hospital, Trinity

College, University of Dublin, Dublin, Ireland; ${ }^{2}$ Trinity Translational Medicine Institute, St James' Hospital, Dublin, Ireland; ${ }^{3}$ Neonatology, Coombe Women and Infants' University Hospital, Dublin, Ireland; ${ }^{4}$ Neonatology, Our Lady's Children's Hospital, Crumlin, Dublin, Ireland; ${ }^{5}$ European Foundation for the Care of Newborn Infants, Munich, Germany and ${ }^{6}$ Section of Neonatal Medicine, Department of Medicine, Imperial College London, Chelsea and Westminster campus, London, UK

Correspondence: Eleanor J. Molloy (eleanor.molloy@tcd.ie)

\section{REFERENCES}

1. United Nations General Assembly Convention on the Rights of the Child, 20 November 1989. Annu. Rev. Popul. Law 16, 485-501 (1989).

2. Bate J., et al Public and patient involvement in paediatric research Archives of Disease in Childhood-Education and Practice. https://doi.org/10.1136/ archdischild-2015-309500

3. Hunter, L. et al. Advancing child health research in the UK: the Royal College of Paediatrics and Child Health Infants' Children's and Young People's Research Charter. Arch. Dis. Child. 102, 299-300 (2017).

4. Ennis, L. \& Wykes, T. Impact of patient involvement in mental health research longitudinal study. Br. J. Psychiatry 203, 381-386 (2013).

5. Molloy, E. J., . \& Gale, C. \& Marsh, M. \& Bearer, C. F. \& Devane, D. \& Modi, N. Developing core outcome set for women's, newborn, and child health: the CROWN Initiative.Pediatr Res 84, 316-317 (2018).

6. Richards, T., . \& Godlee, F. The BMJ's own patient journey. BMJ 348, g3726 (2014).

7. EFCNI, Position Paper: Involvement of parent representatives in neonatal research, 2017; https://www.efcni.org/wp-content/.../04/2017_10_26_Parents_In_ Research_web.pdf 